\title{
Percepción de la calidad de vida en pacientes con cáncer de próstata avanzado: un estudio cualitativo descriptivo
}

\section{Perception of the Quality of Life of Patients with Advanced Prostate Cancer: A Descriptive Qualitative Study}

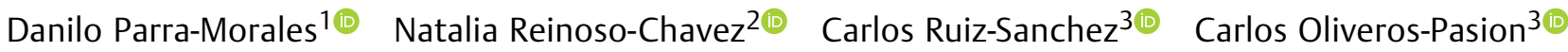 \\ Hector Posso-Valencia2 ${ }^{2}$ Sergio Arrieta-Vera ${ }^{4}$ Marino Cabrera-Fierro ${ }^{3}$ ()
}

${ }^{1}$ Maestrante Epidemiología, Universidad de La Sabana, Chía, Colombia

2 Profesor Cátedra de Medicina, Universidad de La Sabana, Chía, Colombia

3 Médico Urólogo, Departamento de Urología, Hospital Universitario

Dirección para correspondencia Danilo Andrés Parra Morales, MD, Universidad de La Sabana, Chía, Cundinamarca, Colombia de La Samaritana, Bogotá, Colombia

${ }^{4}$ Psicólogo y Comunicador Social, Asistente de Investigación cualitativa, Consultor Externo

Urol Colomb 2021;30(4):e253-e264.

\section{Resumen}

\section{Palabras Clave}

- cáncer De Próstata

- calidad de vida

- entrevista por teléfono

- apoyo social

- bienestar

- estrategias de afrontamiento
Objetivo Describir las percepciones de calidad de vida en una muestra de pacientes con cáncer de próstata avanzado de un hospital universitario.

Métodos Desde un enfoque cualitativo descriptivo, se realizaron entrevistas semiestructuradas y líneas de tiempo para explorar la percepción de calidad de vida de siete pacientes con cáncer de próstata desde su diagnóstico. Por medio de un análisis temático de corte inductivo-deductivo, se identificaron las dimensiones de la calidad de vida que se perciben con mayores cambios a partir de la enfermedad y su tratamiento. Resultados Entre las dimensiones que se encuentran más frecuentemente afectadas están las funciones urinaria, sexual y hormonal. Así mismo, los participantes describen afectaciones relacionadas con los síntomas generales del cáncer y su tratamiento, el estado de ánimo y las limitaciones en la atención por el sistema de salud. Por otra parte, los participantes reportan que su calidad de vida se ve fortalecida gracias a nuevos hábitos y prácticas personales, así como la consolidación del apoyo social, en el que incluyen a la pareja, la familia y la relación con su médico tratante.

Conclusiones El fortalecimiento de la relación médico-paciente basada en el trato cordial, disposición afectiva y empatía al momento de la valoración médica, y la consolidación de una adecuada red de apoyo social como mecanismo de afrontamiento ante el proceso de enfermedad se establecen como las principales estrategias para incrementar la percepción de calidad de vida relacionada con la salud de los pacientes con cáncer de próstata avanzado. recibido

01 de febrero de 2021

aceptado

19 de agosto de 2021
DOI https://doi.org/

$10.1055 / \mathrm{s}-0041-1740269$.

ISSN 0120-789X.

e ISSN 2027-0119.
(C) 2021. Sociedad Colombiana de Urología. All rights reserved. This is an open access article published by Thieme under the terms of the Creative Commons Attribution-NonDerivative-NonCommercial-License, permitting copying and reproduction so long as the original work is given appropriate credit. Contents may not be used for commercial purposes, or adapted, remixed, transformed or built upon. (https://creativecommons.org/ licenses/by-nc-nd/4.0/)

Thieme Revinter Publicações Ltda., Rua do Matoso 170, Rio de Janeiro, RJ, CEP 20270-135, Brazil 


\begin{abstract}
Keywords

- prostate cancer

- quality of life

- phone interview

- social support

- wellness

- coping strategies

Purpose To describe the preception of the quality of life of a sample of patients with advanced prostate cancer from a university hospital.

Methods Based on a descriptive qualitative approach, semi-structured interviews were held and timelines were developed to explore the perception of quality of life of seven patients with prostate cancer since their diagnosis. Through an inductivedeductive thematic analysis, the dimensions of quality of life that have had more changes due to the disease and its treatment were identified.

Results Among the quality of life dimensions that are most frequently affected are urinary, sexual and hormonal functions. Likewise, the participants describe affectations related to the general symptoms of cancer and its treatment, changes in mood, and limitations in the care provided by the health system. However, participants report that their quality of life is strengthened by new habits and personal practices, as well as with the consolidation of social support, in which they include their partners, their families, and their relationship with their treating physician.

Conclusions The strengthening of the doctor-patient relationship based on cordial treatment, affective disposition and empathy at the time of the medical evaluation, and the establishment of an adequate social support network as a coping mechanism in the face of the disease process are consolidated as the main strategies to increase the perception of the health-related quality of life of patients with advanced prostate cancer.
\end{abstract}

\section{Introducción}

A nivel global, una de las neoplasias más prevalentes en hombres es el cáncer de próstata, ${ }^{1}$ una enfermedad más incidente en la población masculina por encima de los 60 años de edad, que alcanza un riesgo acumulado del $4,3 \%$ de ser diagnosticada antes de los 75 años. ${ }^{2}$ Según datos de la Organización Mundial de la Salud (OMS) y de la Agencia Internacional para la Investigación en Cáncer, ${ }^{2}$ en 2018 se presentaron 1.276 .106 nuevos casos y 358.989 muertes por esta patología a nivel global.

Mientras tanto, en Colombia, según la base de datos Globocan, ${ }^{2}$ en 2018, se diagnosticaron 12.712 casos de cáncer de próstata, correspondientes al $12,5 \%$ de los nuevos casos de cáncer del país, lo que ubica a este tipo de enfermedad en un segundo lugar de incidencia, luego del cáncer de seno. Adicionalmente, se reportaron 3.166 muertes por esta patología durante el mismo año, lo que explica el 7,6\% de las muertes por cáncer del país. ${ }^{2}$

Durante el tratamiento y seguimiento de los pacientes con cáncer de próstata, es posible observar la presencia de recaídas o de progresión tumoral, a pesar de que los pacientes hayan recibido tratamientos con fines curativos en fases iniciales de la enfermedad. Esta progresión hacia estadios avanzados se asocia comúnmente a afectaciones en diversos sistemas y, por ende, en la calidad de vida de los pacientes. ${ }^{3}$ Adicionalmente, debemos considerar que los pacientes con progresión tumoral pueden ya haber sido sometidos a múltiples tratamientos a lo largo de su enfermedad, razón por la que algunos de ellos pueden presentar complicaciones o efectos adversos de las intervenciones realizadas previamente, así como la presión psicológica que sienten los enfermos por vivir con un cáncer "incurable". ${ }^{4}$ La suma de todos estos factores genera un impacto negativo significativo en la calidad de vida relacionada con la salud de los pacientes con cáncer de próstata avanzado.

Sin embargo, a pesar de que se conocen estas condiciones, debemos considerar que existen diferentes variables que pueden interferir de manera no intencional en la adecuada interacción y profundidad del interrogatorio al paciente durante la entrevista médica, y entre ellas se describen la duración de la consulta médica, una inadecuada relación médico-paciente, ${ }^{5}$ así como la incomodidad o temor del paciente al momento de relatarle al profesional médico la sintomatología relacionada con la función sexual o con otros temas tabúes para la sociedad. ${ }^{6}$

Lo anterior nos lleva a pensar que, en la actualidad, el paciente podría no estar siendo valorado activa e integralmente en la toma de decisiones con respecto a su propio estado de salud, dejando de lado su opinión con respecto a síntomas, percepciones, sensaciones, sentimientos o tolerancia hacia los diferentes tratamientos propuestos, ${ }^{5}$ lo que favorece que la afectación en la calidad de vida no sea reconocida e intervenida adecuadamente. Por tal motivo, el objetivo principal de este estudio será describir la percepción de los cambios en la calidad de vida de los pacientes con cáncer de próstata avanzado desde el momento inicial del diagnóstico y durante el tratamiento.

\section{Métodos}

\section{Diseño}

Se desarrolló un estudio cualitativo descriptivo, ${ }^{7}$ por medio del cual se describió cómo los pacientes perciben su calidad de 
vida a partir de su experiencia de salud y enfermedad. Lo anterior permitió reconocer cuáles son los elementos que, de acuerdo con los participantes, son determinantes en su calidad de vida, a partir del diagnóstico de cáncer de próstata avanzado. Este diseño de investigación cualitativa, con una larga trayectoria en el campo de la salud, es el método de elección cuando se desean descripciones directas de fenómenos, conocer el qué, quién y dónde de los eventos, y le permite a los investigadores utilizar matices y características de otros métodos de investigación cualitativa que aportan información importante para la práctica.

\section{Participantes}

Se seleccionaron siete pacientes de sexo masculino con diagnóstico de carcinoma de próstata en estadio avanzado. Los participantes provinieron de dos fuentes: pacientes de un hospital universitario en Bogotá, Colombia, y pacientes de un consultorio médico de un urólogo-oncólogo.

La selección de participantes fue por criterio, incluyendo los siguientes: 1) pacientes diagnosticados con cáncer de próstata avanzado quienes acudían a consulta de servicio de urología oncológica, y 2) que estaban recibiendo tratamiento hormonal de primera línea, o eran pacientes resistentes a la castración por tratamiento hormonal, independiente del tratamiento recibido previamente. Por otro lado, se excluyeron pacientes que 1) tuvieran diagnóstico de cáncer de próstata localizado o localmente avanzado, 2) tuvieran compromiso mental debido a metástasis cerebrales, o 3) presentaran un puntaje en la escala del Eastern Cooperative Oncology Group (ECOG) mayor o igual a 3.

\section{Procedimiento}

\section{Método de recolección de información}

Se realizaron entrevistas semiestructuradas y líneas de tiempo. Estas técnicas se aplicaron por medio de conversaciones telefónicas, debido a que, a causa de la emergencia sanitaria por COVID-19, que nos obligó a tomar medidas de aislamiento preventivo, no fue posible realizar estas entrevistas presencialmente. Lo anterior nos llevó a considerar medidas especiales que se describirán en las consideraciones éticas.

La línea del tiempo, como técnica visual que permite la recolección de información, ${ }^{8}$ fue utilizada con el objetivo de que cada participante narrara su historia de vida desde el momento en que se enteró del diagnóstico, e identificara los sucesos significativos que dan cuenta de los determinantes de la calidad de vida en pacientes con este tipo de patología. Lo anterior se realizó tras la formulación de la siguiente pregunta abierta y general: ¿Cuál ha sido su historia personal con esta enfermedad desde que se enteró del cáncer hasta hoy?

Por su parte, la entrevista semiestructurada consistió en una serie de preguntas realizadas al participante, formuladas con base en la historia de vida relatada y en las posibles afectaciones a la calidad de vida identificadas en la revisión de la literatura. Ambas técnicas fueron aplicadas durante la misma sesión, a partir de una conversación telefónica entre participante y entrevistador, que duró entre 40 minutos y 1 hora.

\section{Análisis de información}

La información se analizó con base en los criterios de un análisis temático de corte inductivo-deductivo, que "permite identificar, organizar, analizar en detalle y proporcionar patrones o temas a partir de una cuidadosa lectura y relectura de la información recogida y, así, inferir resultados que propicien la adecuada comprensión o interpretación del fenómeno en estudio". ${ }^{9}$ El proceso de análisis temático se desarrolló a partir de las etapas descritas por Braun y Clarke: ${ }^{9}$

1) Familiarizarse con los datos, lo cual implicó la transcripción y relectura de la información por parte del equipo investigador, con el fin de conocer a profundidad los datos. Lo relatado por los participantes fue estrictamente transcrito por miembros del equipo investigador del proyecto, por medio del programa oTranscribe (Elliot Bentley, MuckRock, US). La transcripción se realizó de manera que quedara plasmada la mayor cantidad de información posible (incluyendo silencios, cambios de actitud o de tono etc.); 2) codificación, en la que se identificaron y etiquetaron asuntos relevantes para los participantes, con el fin de establecer patrones y agrupar segmentos de información similares; cada entrevista fue analizada por medio del programa ATLAS.ti (Scientific Software Development GmbH, US); 3) búsqueda de temas, en la que se analizó cómo los códigos establecidos en la fase anterior se agrupaban para formar temas orientados por las categorías a priori, que pudieran tener relevancia para la pregunta de investigación. 4) revisión de temas, en la que se analizaron los temas previamente identificados, con el fin de evaluar si estos se relacionaban con la teoría propuesta y describían fielmente los relatos de los participantes; 5) definición de temas, en la que se determinaron definitivamente los temas y subtemas, así como su descripción; y, finalmente, 6) la elaboración del informe final de investigación. ${ }^{9}$

\section{Criterios de Rigor}

Los investigadores realizaron triangulación de sus distintas interpretaciones del fenómeno buscando patrones de convergencia ${ }^{10}$ durante todas las fases del análisis de datos. Dos investigadores de diferentes disciplinas realizaron la codificación inicial de todas las entrevistas. Por último, compartieron y discutieron sus codificaciones y llegaron a acuerdos sobre los hallazgos.

Por otra parte, por medio de discusiones acerca de los hallazgos y del progreso de la investigación, se realizó revisión con pares entre el investigador y un par imparcial, con el fin de respaldar la credibilidad y la integridad de los datos. ${ }^{11}$ En este caso, la revisión con pares se realizó con la segunda autora: desde las primeras fases de la investigación, con quien se discutió el diseño metodológico, la recolección de datos, y las diferentes fases del análisis y los resultados.

Por último, durante toda la investigación, se aseguró la auditabilidad como criterio que permite a otro investigador "seguir la pista o la ruta de lo que el investigador original ha hecho". ${ }^{12}$ Para esta investigación, se garantizó la auditabilidad mediante la aplicación de un registro 
continuo de la toma de decisiones metodológicas durante todo el proceso de investigación, con el fin de que cada uno de los pasos ejecutados durante el estudio, así como la razón para la toma de decisiones en las diferentes fases de la investigación, contaran con precisión y transparencia. Este proceso de auditoría es particularmente importante en los estudios cualitativos descriptivos, ya que se recomienda dar detalles metodológicos de manera que el lector pueda examinar la forma en la que se recogió la información y se obtuvieron resultados confiables. ${ }^{13}$

\section{Consideraciones éticas}

Todas las entrevistas contaron con la firma del consentimiento informado de los participantes. Este consentimiento fue socializado inicialmente con cada uno de los participantes, durante la etapa de reclutamiento; en ese momento, se le explicó a los pacientes que su participación sería confidencial, anónima y voluntaria, y que la sesión sería grabada para fines del análisis de la información. Una vez que cada paciente manifestó su deseo de participar en la investigación, se envió el consentimiento informado por correo electrónico, para que este pudiera ser estudiado en detalle y firmado por los entrevistados, quienes enviaron evidencia de la firma del documento a los investigadores. Posteriormente, en una llamada realizada el día anterior a la sesión, se recordó a los participantes las condiciones de su participación y que la entrevista sería grabada.

Adicionalmente, considerando la situación de aislamiento de la población por la pandemia por COVID-19, que dificultó la realización de las entrevistas de manera presencial, se establecieron consideraciones éticas a tener en cuenta a la hora de realizar las entrevistas de forma virtual (telefónica):

1. Durante el reclutamiento, se indagó por la posibilidad de que los participantes contaran, primero, con los elementos necesarios para realizar la entrevista, así como con un espacio en el que puedan sentirse cómodos y con la privacidad suficiente para hablar de su vida íntima, de la enfermedad, y de posibles temas sensibles.

2. La importancia del uso de la línea del tiempo no sólo como estrategia metodológica para la recolección de la información, sino también como herramienta para hacer menos intrusiva y directiva la entrevista, en especial con aquellos temas sensibles, ya que los participantes son quienes relatan de manera voluntaria las afectaciones que mayormente influyen en su calidad de vida.

3. Toma de conciencia respecto al establecimiento del rapport. En la fase de apertura de la entrevista, se dedicó un tiempo prudente al establecimiento de rapport con el entrevistado, pues si bien la empatía y la generación de confianza entre el participante y el entrevistador puede surgir de forma natural, el hecho de que la entrevista se realice de forma virtual o telefónica podía dificultar este proceso y limitar la comodidad del entrevistado de brindar determinada información. ${ }^{14} \mathrm{Si}$ bien se reconoce que al realizar entrevistas de manera telefónica puede no identificarse información no verbal, durante la entrevista se prestó atención a silencios, pausas y cambios en el tono de los entrevistados que pudieran aportar significados que no se expresaran verbalmente. Para compensarlo, se establecieron preguntas adicionales que se describen en el siguiente punto.

4. Se estableció una batería robusta de preguntas auxiliares, en la que se buscó indagar por cada una de las categorías y subcategorías, con el fin de profundizar en los relatos de los participantes y explorar posibles temas identificados en la literatura y que no surgieran espontáneamente durante la entrevista.

\section{Resultados}

\section{Características de los participantes}

Todos los participantes tenían edades entre los 53 y los 65 años, con un promedio de 60,8 años. Además, debido a que, durante el proceso de selección, se buscaron participantes con características variadas, los pacientes entrevistados se diferenciaron por el tipo de régimen de afiliación al sistema de salud (pues se incluyeron sujetos de los regímenes subsidiado, contributivo y medicina prepagada), el tipo de tratamiento que recibieron durante la trayectoria de su enfermedad, y el tiempo transcurrido desde el diagnóstico hasta el momento de la entrevista (que osciló entre 1,5 y 4,5 años). La información de las características de los participantes se expresa a continuación, en la - Tabla 1.

\section{Resultados por categorías}

Como resultado, se obtuvieron 9 categorías (5 a priori, o identificadas a partir del marco teórico, y 4 emergentes, provenientes de los relatos de los participantes), que se dividieron en 24 subcategorías. Estas categorías y subcategorías se muestran en la - Tabla 2.

En términos generales, los participantes describieron cambios en su calidad de vida relacionados con los síntomas urinarios, con las alteraciones en las funciones sexual y hormonal que se producen tras los tratamientos, y con las afectaciones generales relacionadas con el cáncer, incluyendo el tratamiento del mismo; también señalaron cambios en su calidad de vida asociados al estado de ánimo. Adicionalmente, los participantes relataron afectaciones a la calidad de vida vinculadas al funcionamiento del sistema de salud y a la imposibilidad de realizar ciertas actividades. Por otro lado, los participantes también describieron cambios en su calidad de vida que favorecen su bienestar, relacionados a la atención médica, a las estrategias de afrontamiento que tomaron ante el diagnóstico, y a la red de apoyo social. A continuación, se describen los resultados obtenidos por cada categoría.

\section{Afectación a la calidad de vida en pacientes relacionada con síntomas urinarios}

En primer lugar, los participantes describieron algunas afectaciones a la calidad de vida asociadas a la presencia de síntomas urinarios. $\mathrm{Al}$ respecto, los pacientes reportaron 
Tabla 1 Características de los participantes del estudio

\begin{tabular}{|l|l|l|l|l|}
\hline Participante & $\begin{array}{l}\text { Años transcurridos } \\
\text { desde el } \\
\text { diagnóstico }\end{array}$ & $\begin{array}{l}\text { Tipo de } \\
\text { metástasis }\end{array}$ & Tipo de tratamiento & $\begin{array}{l}\text { Tipo de régimen } \\
\text { de afiliación }\end{array}$ \\
\hline 1 & 4 & $\begin{array}{l}\text { Pulmonar y } \\
\text { ósea }\end{array}$ & Quimioterapia y orquidectomía & Subsidiado \\
\hline 2 & 2 & No reporta & $\begin{array}{l}\text { Prostatectomía, quimioterapia, y tratamiento } \\
\text { hormonal }\end{array}$ & Contributivo \\
\hline 3 & 1,5 & No reporta & Tratamiento hormonal & Medicina prepagada \\
\hline 4 & 2,5 & Ósea & Tratamiento hormonal & Contributivo \\
\hline 5 & 4,5 & No reporta & $\begin{array}{l}\text { Prostatectomía, tratamiento hormonal, y } \\
\text { orquidectomía }\end{array}$ & Contributivo \\
\hline 6 & 1,6 & Ósea & Tratamiento hormonal & Subsidiado \\
\hline 7 & 3 & Ósea & Tratamiento hormonal y orquidectomía \\
\hline $\begin{array}{l}\text { Características de los participantes de acuerdo con el tiempo transcurrido desde el diagnóstico, el tipo de metástasis presentada, el tipo } \\
\text { de tratamiento y el tipo de régimen de afiliación al sistema de salud. }\end{array}$
\end{tabular}

haber vivido episodios de incontinencia, que aparecieron como un efecto adverso de las intervenciones médicas realizadas, aunque no fue una afectación narrada con frecuencia.

Por el contrario, algunos participantes manifestaron que el dolor, asociado tanto a síntomas urinarios irritativos como a síntomas urinarios obstructivos, sí representa una afectación significativa. Sobre esto, el Participante 5 afirmó: "hay veces que me arde mucho para orinar, se me dificulta a veces, me da como si estuviera yo tapado, [...] la parte de la infección urinaria me afecta un poco el día a día, que me duerma, uno dice que va a orinar y le entra el malestar de la espalda y me duele". En la misma línea, frente a los síntomas urinarios obstructivos, los entrevistados afirmaron sentir dificultad al orinar, lo que se caracteriza en los relatos por la disminución del calibre de la orina y por la necesidad de "hacer un esfuerzo para evacuar".

Adicionalmente, algunos participantes afirmaron que, debido al número de veces que se levantan a orinar en las noches, el sueño se ve interrumpido de manera constante.

\section{Afectación a la calidad de vida en pacientes con alteración en la función sexual}

Algunos pacientes reportaron afectaciones a la calidad de vida relacionadas con cambios en su vida sexual. Entre los deterioros descritos, se destaca la disminución del deseo sexual y la imposibilidad para tener erecciones. Por ejemplo, el Participante 7 contó: “en ese sentido, la vida cambió mucho, porque no he vuelto a sentir nada. Desde que me operaron y la enfermedad, no funciona ni nada, se la pasa es "dormido". No sé qué hacer para eso. No siento nada".

Además, la afectación en la función sexual - en el caso de los entrevistados - se extiende a dimensiones relacionadas con la percepción de la masculinidad y de los roles de género. Por ejemplo, los participantes expresaron inconformidad con ellos mismos y tristeza por el hecho de no poder complacer sexualmente a sus parejas, o se refieren a cambios en el rol de autoridad de la familia, que asocian a la "pérdida de la hombría”. Tal es el caso del Participante 6, que afirmó lo siguiente: "la mujer mía todavía está joven y, a pesar de eso, por eso no hay problema con ella, pero pues para uno como hombre sí a veces se siente mal".

\section{Afectación a la calidad de vida en pacientes con alteraciones en la función hormonal}

Por su parte, los participantes que recibieron tratamiento hormonal reportaron afectaciones asociadas a la función hormonal. Entre estas, se resaltan aquellas relacionadas con los cambios en la forma del cuerpo, como aumento de peso, aumento del perímetro abdominal, y presencia de ginecomastia, que, de acuerdo con los entrevistados, representan una molestia en la vida cotidiana.

También los pacientes reportaron sentir permanentemente cambios bruscos en el estado de ánimo, generalmente orientados hacia la rabia o la agresividad.

Adicionalmente, los participantes dijeron percibir afecciones o molestias por las oleadas de calor en el cuerpo, que producen incomodidad, sudoración $\mathrm{y}$, en algunas ocasiones, interrumpen el sueño. Para algunos participantes, esta consecuencia del tratamiento llegó a ser descrita como un elemento que les afecta en gran medida y de manera frecuente. Al respecto, el Participante 3 relató lo siguiente: lo que más me afecta es el calor. Me sube de pronto... estoy tranquilo y, de pronto, empiezo a sentir en el cuerpo algo, cómo empieza como por el estómago, como un síntoma así que siento como que me va a dar algo, y empiezo a sentir el calor $y$ me sube hasta la cabeza, y sudo hartísimo. A veces me da, al día, diez veces; en la noche me da más que de día. Vale la pena mencionar que, de acuerdo con los participantes, estos episodios de oleadas de calor - especialmente los que se presentan en la noche - pueden afectar la conciliación del sueño de los participantes o despertarlos durante la madrugada. 
Tabla 2 Categorías y subcategorías

\begin{tabular}{|c|c|c|}
\hline Categorías & Subcategorías & Densidad \\
\hline \multicolumn{3}{|l|}{ Categorías a priori } \\
\hline \multirow{3}{*}{$\begin{array}{l}\text { Afectación a la calidad de vida en } \\
\text { pacientes relacionada con síntomas } \\
\text { urinarios }\end{array}$} & Presencia de incontinencia urinaria & 2 \\
\hline & Sueño interrumpido por la necesidad de ir al baño & 2 \\
\hline & Presencia de síntomas urinarios obstructivos e irritativos & 12 \\
\hline \multirow{2}{*}{$\begin{array}{l}\text { Afectación a la calidad de vida en } \\
\text { pacientes relacionada con la función } \\
\text { sexual }\end{array}$} & Afectación de la sexualidad & 9 \\
\hline & $\begin{array}{l}\text { Percepción de pérdida de la masculinidad, del rol de género, y } \\
\text { de la persona en su entorno social }\end{array}$ & 3 \\
\hline \multirow{3}{*}{$\begin{array}{l}\text { Afectación a la calidad de vida en } \\
\text { pacientes relacionada con alteraciones en } \\
\text { la función hormonal }\end{array}$} & Afectación por cambios en la forma o apariencia del cuerpo & 4 \\
\hline & Cambios de humor, ira, o irritabilidad & 2 \\
\hline & Incomodidad por calores en el cuerpo & 11 \\
\hline \multirow{3}{*}{$\begin{array}{l}\text { Afectación a la calidad de vida relacionada } \\
\text { con síntomas generales en cáncer y su } \\
\text { tratamiento }\end{array}$} & Dolor relacionado con la enfermedad y/o con los tratamientos & 35 \\
\hline & Fatiga & 17 \\
\hline & Náusea, pérdida de apetito, y mareo & 8 \\
\hline \multirow{3}{*}{$\begin{array}{l}\text { Afectación a la calidad de vida relacionada } \\
\text { con el estado de ánimo y la salud mental }\end{array}$} & Sentimientos de inutilidad o de carga familiar & 9 \\
\hline & $\begin{array}{l}\text { Afectaciones a la calidad de vida por emociones negativas } \\
\text { alrededor del diagnóstico }\end{array}$ & 12 \\
\hline & Afectaciones por temor al COVID- 19 & 6 \\
\hline \multicolumn{3}{|l|}{ Categorías emergentes } \\
\hline \multirow{2}{*}{$\begin{array}{l}\text { Afectación a la calidad de vida por no } \\
\text { poder realizar actividades significativas }\end{array}$} & Imposibilidad para realizar deporte o actividades lúdicas & 20 \\
\hline & Imposibilidad para trabajar & 4 \\
\hline \multirow{2}{*}{$\begin{array}{l}\text { Afectación a la calidad de vida relacionada } \\
\text { con el funcionamiento del sistema de } \\
\text { salud }\end{array}$} & $\begin{array}{l}\text { Afectación a la calidad de vida relacionada con la labor del } \\
\text { personal de salud }\end{array}$ & 16 \\
\hline & $\begin{array}{l}\text { Afectación a la calidad de vida por limitación de acceso a los } \\
\text { servicios del sistema de salud }\end{array}$ & 22 \\
\hline \multirow{3}{*}{$\begin{array}{l}\text { Calidad de vida favorecida por el } \\
\text { fortalecimiento de prácticas personales }\end{array}$} & Incorporación de nuevos estilos de vida saludables & 5 \\
\hline & $\begin{array}{l}\text { Espiritualidad y religiosidad como determinantes del } \\
\text { bienestar en salud }\end{array}$ & 6 \\
\hline & $\begin{array}{l}\text { Estrategias de afrontamiento frente a la enfermedad que } \\
\text { generan bienestar y relacionadas con "una actitud positiva" }\end{array}$ & 13 \\
\hline \multirow[t]{3}{*}{$\begin{array}{l}\text { Calidad de vida favorecida por buen apoyo } \\
\text { social }\end{array}$} & $\begin{array}{l}\text { Adaptación y entendimiento por parte de la pareja ante los } \\
\text { cambios en la función sexual }\end{array}$ & 5 \\
\hline & $\begin{array}{l}\text { Aumento en la calidad de vida por red de apoyo familiar sólida } \\
\text { y atención familiar }\end{array}$ & 22 \\
\hline & $\begin{array}{l}\text { Atención médica como determinante para el bienestar en la } \\
\text { calidad de vida }\end{array}$ & 24 \\
\hline
\end{tabular}

\section{Afectación a la calidad de vida relacionada con síntomas generales del cáncer y su tratamiento}

Por otro lado, los entrevistados describieron afectaciones relacionadas con la presencia de síntomas que son comúnmente manifestados por los pacientes con cáncer, tales como dolor, ya sea generado por la enfermedad en sí o secundario a los tratamientos realizados, fatiga, pérdida de apetito, insomnio de conciliación, mareo y náuseas.

Entre las afectaciones mencionadas anteriormente, el dolor fue reportado en múltiples ocasiones por todos los participantes como un síntoma que afecta de manera frecuente su vida cotidiana. De hecho, el dolor, tanto el que está relacionado con los síntomas de la enfermedad en sí como el que es consecuencia de los tratamientos o intervenciones médicas, fue de las afectaciones más comúnmente referidas. Sobre esto, el Participante 7 relató: "al principio, cuando no sabía que era la enfermedad, era un dolor muy intenso; no podía dormir, me levantaba en la noche".

Además, los entrevistados también afirmaron sentir dolor inmediatamente después de la aplicación del medicamento, o los días posteriores a la misma. De hecho, los días inmediatamente posteriores a los tratamientos fueron descritos por algunos pacientes como los momentos en los que más percibieron afectaciones a la calidad de vida. Por ejemplo, el Participante 1 relató: después de iniciar las 
"quimios", "creo que cuando comenzó a obrar lo que es el medicamento, pues las partes afectadas o en las que siempre tuve dolencia siempre, pues ahí me afectó más". Adicionalmente, de acuerdo con los pacientes, el dolor también se relaciona con las afectaciones del sueño, pues los participantes reportaron la dificultad para poder conciliar el mismo y el hecho de despertarse frecuentemente en la madrugada, debido a dolores fuertes. Sobre esto, el Participante 5 refirió: "a veces, el dolor en las piernas, me coge mucho malestar en la pierna izquierda de la rodilla para arriba, que es un dolor que inclusive me despierta en la noche".

Por su parte, la fatiga fue otro de los síntomas relacionados con cáncer que fue asociado a la percepción de calidad de vida de los participantes. La fatiga fue relatada como una percepción de "cansancio" o "agotamiento" al realizar actividades cotidianas, o como una consecuencia adversa de los tratamientos. Junto al dolor, la fatiga fue de las afectaciones reportadas más frecuentemente por los entrevistados. Sin embargo, los participantes relataron también elementos como la pérdida de apetito, la sensación de mareo, y náuseas.

\section{Afectación del estado de ánimo y la salud mental}

Los participantes describen afectaciones relacionadas con alteraciones del estado de ánimo que han vivido durante la trayectoria de su enfermedad. En esta categoría, se definieron tres subcategorías denominadas "sentimientos de inutilidad o de ser una carga para la familia", "afectaciones a la calidad de vida por emociones negativas alrededor del diagnóstico", y "afectaciones por temor a contagiarse de COVID-19".

Respecto a los sentimientos de inutilidad o de percibirse como una carga familiar, los participantes refirieron sentir que los cuidados que la familia brinda son un esfuerzo adicional de parte de ellos; también afirmaron que, al dejar de trabajar, se sienten "inútiles", o como "una carga tanto para los familiares como para la sociedad". En ese sentido, el Participante 6 afirmó: para ellos [la familia] no es carga, yo sé. Pero, por ejemplo, no me pueden dejar sólo; ellos viven muy pendientes, que el medicamento a cuáles horas, que esto, que la fruta. Entonces, yo digo: "antes no hacían todo esto, ¿por qué ahora lo tienen que hacer?”. Es ahí donde yo veo que es como una carga.

Por otro lado, algunos entrevistados hicieron referencia a las emociones negativas que surgen, especialmente en el momento del diagnóstico, entre las que predominan la frustración, la tristeza y el miedo, tanto a la muerte como al nuevo proceso de tratamiento. En ese sentido, los participantes describieron cómo, al momento de enterarse del diagnóstico o en las etapas iniciales de la enfermedad, se sentían tristes y percibían que su proyecto de vida había cambiado para mal. El temor a la muerte y a enfrentar el nuevo proceso de tratamiento por la enfermedad también es otro hallazgo relevante en relación con las afectaciones de la calidad de vida.

Así mismo, el temor de los participantes no es únicamente ante el proceso de enfermedad que están viviendo; algunos de ellos describieron afectaciones a la calidad de vida que provienen de la pandemia por COVID-19, pues los pacientes afirman sentir temor por ser más vulnerables ante el contagio.

\section{Afectación a la calidad de vida por no poder realizar actividades significativas}

Los participantes reportaron afectaciones a la calidad de vida asociadas a no poder realizar las actividades lúdicas y deportivas que realizaban antes del diagnóstico, lo cual describieron como cambios drásticos en sus vidas a raíz de la enfermedad. En este sentido, el Participante 4 narró lo siguiente: "debido a eso [la enfermedad] pues eh, no he vuelto a hacer deporte, digamos a correr, lo que yo siempre hacía; digamos la media maratón y todas esas cosas, ya no las volví a hacer. Por ejemplo, yo trotaba todos los días, ya no lo volví a hacer".

En esta categoría, adicionalmente se encontró que la calidad de vida se puede ver afectada desde el punto de vista laboral, ya sea por despido laboral posterior al diagnóstico o por no tener el mismo rendimiento en el trabajo. Dejar de trabajar se refleja, naturalmente, en una disminución en los ingresos de los participantes, lo cual genera preocupación y afectación en la calidad de vida en algunos de los sujetos, debido a que, por el hecho de no poder trabajar o de haber sido pensionado anticipadamente, su capacidad económica se ve disminuida.

\section{Afectación a la calidad de vida relacionada con el funcionamiento del sistema de salud}

Entre los hallazgos relevantes de esta investigación está la percepción que tienen los participantes de afectaciones a la calidad de vida a causa de asuntos relacionados con el sistema de salud. Sobre esto, se reportan tanto elementos relacionados con las limitaciones en la accesibilidad a los servicios que presta el sistema de salud, debido a los trámites administrativos que son necesarios, como aspectos relacionados con la labor del personal de salud.

Frente a esto último, algunos pacientes afirmaron que, desde su percepción, su calidad de vida y salud se afectaron, debido a la mala relación o malos tratos por parte de los profesionales de la salud que les atendían. Por ejemplo, el Participante 2 narró lo siguiente: "Porque el día que tuve la primera cita con la señora que nos fue a autorizar de oncología, sí ahí se me bajó el ánimo, mejor dicho, al piso, tenaz [...] y le dije a mi señora: 'cambiemos de oncólogo, porque esta señora sí me tiene traumatizado', y entonces eso lo desmoraliza a uno mucho, porque un oncólogo que le llegue a decir a uno eso, hable mal [del tratamiento], y después diga que sí está bien lo que se ha hecho, entonces no entiende uno. ¿Para qué habla?, ¿para qué dice cosas que no son? Eso le queda a uno en la cabeza".

Algunos participantes también relataron afectaciones relacionadas con lo que definieron como "mala atención médica", refiriéndose a supuestas omisiones o negligencias por parte de los profesionales que los atendieron. Al respecto, los participantes relataron principalmente demoras en la realización de exámenes diagnósticos a pesar de, según ellos, tener síntomas claros; así mismo, la percepción de que no se 
prescribieron adecuadamente medicamentos para su enfermedad es la queja más común de los pacientes.

Por su parte, los participantes también relataron afectaciones relacionadas con las limitaciones de acceso a la salud, debido al exceso de trámites que solicita el sistema. Los pacientes reportaron que, debido a cómo funciona el sistema de salud, se presentan demoras en el acceso a los servicios médicos, hay dificultad para encontrar los profesionales especializados, y que, cuando hay cambios en los convenios entre las instituciones, eso se traduce en obstáculos para continuar con el tratamiento o acceder a los servicios de salud. Por ejemplo, el Participante 2 dijo: "entonces ya tenía programada una cirugía en la, en la Clínica, ya tenía los exámenes, tenía radiografías, tenía todo lo que me habían pedido, y ya el Doctor, allá del Centro Médico, ya me había hecho todos los exámenes, ya teníamos todo listo [...]. Y, en ese momento, VIDA EPS [pseudónimo] acaba el convenio con el Centro y quedé en el limbo; entonces, no me pudieron hacer la operación".

Además, los pacientes también refirieron limitaciones al acceso al sistema de salud que han surgido a causa del COVID-19.

\section{Calidad de vida favorecida por el fortalecimiento de prácticas personales}

Por otro lado, los participantes no sólo describieron elementos que perjudican su calidad de vida, sino que hicieron referencia a los elementos o comportamientos que la mejoran y generan bienestar. Entre estos generadores de bienestar se destacan la incorporación de nuevos estilos de vida saludables, las estrategias de afrontamiento descritas como "tener una actitud positiva", y la espiritualidad - o religiosidad - como determinante en la salud.

Frente a la adopción de hábitos de vida saludables, los entrevistados reportaron cómo han adquirido rutinas de alimentación que mejoran su calidad de vida desde que fueron diagnosticados con la enfermedad. Por ejemplo, el Participante 2 comentó: "Eso depende de la comida de uno; yo trato de comer alcalino y dejar las grasas, esas saturadas que dicen. Y, por ahí de vez en cuando, pues me pego una limpieza con el ajo y estas cuestiones medicinales, todo medicinal, tratando de, pues como ya me están metiendo tanto fármaco, pues tratar de que todo sea natural para que el organismo trabaje bien". Algunos pacientes también relataron haber dejado hábitos potencialmente nocivos; ese es el caso del Participante 2, que afirmó lo siguiente: "dejé de fumar, eso sí. Me dio un poquito duro, pero, bueno ya, ya, ya superé esa etapa, y ya no me fumo nada, ya no siento ganas de fumar".

Adicionalmente, se refirieron a las estrategias de afrontamiento que generan bienestar. Sobre eso, los pacientes relatan cómo tener una "actitud positiva" frente a la enfermedad es determinante para mejorar la calidad de vida y el estado de ánimo. Por ejemplo, el Participante 5 narró: "Eso es lo que me ha mantenido con ánimos, con alegría, yo no me pongo a pensar en que estoy enfermo, que me pasó esto o aquello, no me concentro en esas cosas; más bien, me levanto con otra actitud: hay que hacer esto, esto, tenemos la cita hoy". De igual forma, algunos de los entrevistados resaltaron cómo la espiritualidad y la religión se constituyen como una herramienta para enfrentar la enfermedad y para mejorar la calidad de vida. En ese sentido, el Participante 2 afirmó lo siguiente: "Pues uno se aferra a algo espiritual, católico, pues yo soy católico, y me aferro a Dios, algo grande que le dé a uno energía para seguir adelante. Dar las gracias porque todo lo importante queda, va saliendo bien y sigue uno adelante, ahí va saliendo uno al otro lado".

\section{Calidad de vida favorecida por un buen soporte social} Por último, los participantes describieron generadores de bienestar asociados al "apoyo" brindado por la familia. Entre estos determinantes, resultó llamativa la subcategoría denominada "Adaptación y entendimiento por parte de la pareja ante los cambios en la función sexual", que fue relatada por los entrevistados como esencial para afrontar las consecuencias en la vida sexual derivadas de los tratamientos. Los participantes reportaron los mecanismos de afrontamiento y adaptabilidad que las parejas de los pacientes han tenido al entender y apoyar estos nuevos cambios en su intimidad, y afirmaron que estos cambios son determinantes en su calidad de vida, porque se sienten comprendidos por sus parejas. Sobre lo anterior, el participante 5 afirmó lo siguiente: "Esa parte es muy bonita. Tiene 2 partes: primero, el hecho de que uno es hombre y le hacen la orquidectomía, entonces, "ya no es hombre", pero la verdad es que no es así. La relación sexual no queda funcionando igual, no hay erección, si esa es la pregunta, no hay penetración de ninguna forma, no hay nada. Pero sí hay mucho amor, que es lo que da la vuelta a ese tema. En el caso mío, ya es un giro, de esa relación sexual, donde siempre hay penetración, orgasmos, eyaculación, para el caso mío, se convirtió en amor. Ella lo ha aceptado con total normalidad, ha sido muy bonito, hasta nos hemos inventado cosas, estamos juntos [...] sólo estamos los dos, $y$, por medio de caricias y eso, ella me ha aceptado, como han venido sucediendo las cosas. Para mí eso ha sido muy bonito, porque se ha convertido más en amor. Se ha convertido en algo diferente, en amor, eso ha sido algo positivo".

Asimismo, los participantes también se refirieron a los diferentes apoyos que sus familiares les brindan en el marco de la enfermedad, que varían desde acompañamiento emocional, comprensión frente a los síntomas, o al hecho de que los familiares se convierten en cuidadores de los pacientes. Según los entrevistados, estos apoyos son determinantes para la calidad de vida. Al respecto, el Participante 3 relató: "siempre me acompañaban mis dos hijas y mi esposa a todo eso, siempre tuve el acompañamiento de ellas, que eso para uno es importantísimo, porque en la parte anímica le ayuda a uno muchísimo sentirse acompañado".

Finalmente, los participantes relataron cómo la atención médica también es un determinante para el bienestar. Esta categoría se entiende como contrapuesta a la descrita anteriormente, según la cual la "mala atención médica 
afectaba la calidad de vida". Al respecto, los participantes reconocieron en la atención médica un generador de mejor calidad de vida. Algunos de los pacientes afirmaron que los tratamientos y las intervenciones a las que fueron sometidos los hicieron sentir mejor en relación a los síntomas; también algunas veces se lo atribuyeron al conocimiento o trato por parte de los médicos que los atienden o a la cobertura de medicamentos por parte del seguro.

Sobre eso, el Participante 4 dijo: "en todo caso, ese doctor, para qué, ese doctor lo atiende, todo lo que me dice yo lo hago, tómese esta droga a las 7 a.m., a las 7, yo me la tomo; si me dice a la 1am, a la 1 a.m. yo me la tomo [...]. Yo sí estoy muy agradecido primero con Dios, y, segundo, a él, que me ha ayudado mucho, $y$ he salido adelante". Sobre este mismo tema, el Participante 2 relató específicamente como la buena atención médica había impactado positivamente su calidad de vida: "Entonces pues, uno, yo le agradezco mucho al Dr. Padilla [pseudónimo] y al Dr. Estrada [pseudónimo], porque, pues, me ayudaron a prolongar la vida, $y$ me dan un poquitico más de calidad de vida [...] porque de todas maneras la extracción de la próstata fue muy buena, y los ganglios y las vesículas que me sacaron fue demasiado bien, $y$, pues, le da un poquitico más de calidad de vida".

\section{Discusión}

En la percepción de la calidad de vida de los hombres con cáncer de próstata avanzado descritos en el estudio, ellos comparten elementos identificados previamente en la literatura centrados en las afectaciones, pero arrojan particularidades sobre las afectaciones priorizadas. Adicionalmente, el estudio describe temas emergentes alrededor de la calidad de vida y las condiciones que pueden favorecerla, particularmente en el estado avanzado.

En primer lugar, entre los cambios reportados por los participantes, y que coinciden con las afectaciones descritas en la literatura, se encuentran las afectaciones a la calidad de vida por síntomas generales del cáncer y su tratamiento. Tal y como lo describe la literatura, ${ }^{15,16}$ los participantes reportaron que el dolor, la fatiga, así como las sensaciones de náuseas y pérdida de apetito son cambios que afectan en gran medida su calidad de vida. Sobre esto, el dolor fue de las afectaciones reportadas más comúnmente por los participantes, que mencionaron esta alteración como consecuencia tanto del cáncer en sí como de los tratamientos para combatirlo.

Por su parte, los pacientes con tratamientos hormonales refirieron afectaciones relacionadas con la función hormonal; de las descritas por Wilde et al. ${ }^{17}$-cambios en la función hormonal que producían reducción o abolición del deseo sexual, disfunción eréctil, oleadas de calor, y cambios en la forma del cuerpo -, los participantes hicieron énfasis en la presencia de calores, relacionándolos además con alteraciones en el sueño.

De las afectaciones en la función sexual descritas por Wilde et al., ${ }^{17}$ los participantes hicieron énfasis en incapacidad de tener erecciones y la pérdida del deseo sexual; sin embargo, en los reportes de los participantes, no hubo alteraciones asociadas a cambios en la eyaculación ni a la evitación de la relación sexual. De hecho, algunos reportaron nuevas dinámicas en la relación sexual con su pareja que describieron como generadores de bienestar. Algunos participantes reportaron el sentimiento de pérdida de la masculinidad, al que se refirieron como una inconformidad consigo mismo por no satisfacer sexualmente a sus parejas y por percibir pérdida de autoridad; este tipo de afectación asociada a la función sexual también fue descrita por Wilde et al. ${ }^{17}$ Los participantes sólo relataron afectaciones a la calidad de vida relacionadas a la función sexual una vez que se les preguntó directamente por ello durante la entrevista semiestructurada, y no por iniciativa propia, lo que indica que las consecuencias en la vida sexual de los pacientes con cáncer de próstata es un tema incómodo y difícil de reportar.

Los síntomas urinarios, e irritativos u obstructivos, son ampliamente descritos en la literatura, ${ }^{18}$ siendo la incontinencia un elemento recurrente en la evaluación de la calidad de vida en pacientes con cáncer de próstata; sin embargo, aunque los participantes reportaron algunos síntomas urinarios, no reportaron este síntoma como una afectación particularmente importante en su calidad de vida.

Por otra parte, los participantes sí reportaron afectaciones a la calidad de vida por cambios en el estado de ánimo, particularmente durante las primeras etapas del diagnóstico. Coincidiendo con la literatura, reportaron sentir "ser una carga familiar". ${ }^{18}$ Sin embargo, los participantes describieron cómo el apoyo de su red familiar y las estrategias de afrontamiento mejoraron su estado de ánimo con el tiempo, elemento central de la discusión que se ampliará más adelante.

Debido al contexto global secundario a la pandemia por COVID-19, algunos participantes describieron sensación de temor al contagio, reconociéndose como pacientes vulnerables por sus antecedentes de cáncer, lo que además también limita la posibilidad de salir de su hogar a realizar actividades diferentes. Frente a ello, es importante dar atención a estos pacientes considerando lo señalado por Ramírez et al., ${ }^{19}$ quienes identifican la emergencia de problemas de salud mental a raíz de la pandemia por COVID-19, con un mayor impacto en personas con preexistencias, personas enfermas, y sus familias. Estos cambios mentales, tales como depresión, ansiedad y temor, están principalmente explicados por la presencia del aislamiento social y la movilidad restringida generada por la pandemia. ${ }^{19}$

El presente estudio descriptivo identifica temas emergentes sobre la calidad de vida de los pacientes con cáncer de próstata avanzado que abren nuevos escenarios para la investigación en el área.

En primer lugar, los participantes reportaron frecuentemente afectación en la calidad de vida por no poder realizar actividades significativas, tales como actividades lúdicas, deportivas, e incluso laborales. Al respecto, uno de los participantes que fue excluido de su trabajo describió haberse visto particularmente afectado por la falta de "compasión" que percibió hacia él por parte de su 
empresa, lo que le generó tristeza y sensación de desesperanza. Y es que, de acuerdo con Vicente et al.,20 “el cáncer genera gran impacto en los costes directos para el trabajador en su tratamiento, costes destinados en apoyo a su cuidado, y un elevado riesgo de no retorno al trabajo y de exclusión social"; y cuando esta exclusión laboral se genera, sobrevienen dificultades económicas o financieras que se relacionan con la presencia de alteraciones del estado del ánimo, ánimo depresivo, y sensación de inutilidad. ${ }^{20}$

En segundo lugar, la mayoría de los participantes reportaron afectación a la calidad de vida relacionada con el funcionamiento del sistema de salud. Si bien no se encontraron estudios relacionados con el impacto del sistema de salud en la calidad de vida de pacientes con cáncer de próstata avanzado específicamente, Giraldo y Ceballos $^{21}$ describieron una serie de barreras del sistema de salud colombiano identificadas para un diagnóstico y tratamiento oportuno en pacientes con cáncer de mama, entre las que se encuentran: 1) el estatus de afiliación al que pertenezcan en el sistema de salud; 2) el papel que desempeña el personal de salud, principalmente en relación con barreras en la comunicación y el tiempo corto de la consulta médica; 3) poca información brindada por el personal de salud con respecto a la enfermedad; y 4) negación de los exámenes diagnósticos y tratamientos, y excesivos trámites para las autorizaciones de los mismos. ${ }^{21}$ Según Peralta, ${ }^{5}$ la atención médica y la relación médicopaciente son un pilar fundamental en la percepción de calidad de vida por parte del paciente, ya que, durante la consulta médica, las interacciones que se produzcan van a influir en los resultados obtenidos. En la entrevista, los participantes relataron cómo su calidad de vida se vio afectada negativamente debido a la relación que llevaban con los médicos y su comportamiento en ocasiones hostil hacia ellos en el momento de la atención médica. Este es un determinante que afecta no sólo la percepción de calidad de vida sino la percepción del estado de salud de los pacientes.

Es importante aclarar que los participantes también describieron la atención médica como generador de bienestar, que mejora la percepción de calidad de vida no sólo asociada a los efectos positivos de los tratamientos, sino al bienestar generado por la relación entre ellos y el personal de salud; de hecho, este tema emergente se contrapone a las afectaciones encontradas en la literatura y compartidas por algunos pacientes, que describen cómo la "mala" atención médica y una relación médico-paciente inadecuada pueden producir daños a la calidad de vida de los pacientes. ${ }^{22}$ Cuando las interacciones médico-paciente se caracterizan por la comunicación efectiva, 1) generan mayor confianza; 2) disminuyen el estrés emocional; 3) favorecen el proceso de diagnóstico y la toma de decisiones durante la consulta médica; 4) mejoran los resultados en salud; 5) disminuyen el número de problemas médico-legales; 6) incrementan la adherencia al tratamiento y a las recomendaciones médicas por parte del paciente; y 7) son un generador de satisfacción en ambas partes (paciente y médico). ${ }^{5}$ Estos elementos reconocidos en la literatura cobran especial relevancia frente a lo referido directamente por los pacientes de cáncer de próstata avanzado entrevistados como factor importante para aumentar o disminuir considerablemente su calidad de vida.

En tercer lugar, los participantes reportaron elementos, prácticas o condiciones que favorecieron la calidad de vida, lo cual no se encuentra en la literatura revisada referida específicamente a los pacientes con cáncer de próstata avanzado, aunque sí en la literatura de pacientes con otros tipos de cáncer, lo que señala la importancia de tener una dieta saludable, la práctica de ejercicio físico, y la suspensión del hábito de fumar como un factor que incrementa el bienestar emocional y la confianza, que, a su vez, favorece la presencia de pensamientos positivos sobre el pronóstico de su enfermedad. ${ }^{23}$ Del mismo modo, las prácticas espirituales y las estrategias de afrontamiento que cada individuo desarrolla ante el diagnóstico fueron elementos que favorecieron el bienestar y la calidad de vida.

Los participantes consideraron que los nuevos estilos de vida saludables son una estrategia para mitigar el impacto de las afectaciones asociadas a los síntomas generales del cáncer y a los efectos adversos de los tratamientos; habiendo reconocido las alteraciones causadas por la enfermedad y las intervenciones médicas, fortalecer nuevos hábitos que promuevan la salud puede ser una de las herramientas que tienen los pacientes para mejorar su calidad de vida. ${ }^{23}$ Por su parte, las estrategias de afrontamiento y las prácticas espirituales generan bienestar relacionado con mejoras en el estado de ánimo, como también se reporta en la literatura. ${ }^{24}$ Las prácticas descritas en este tema, así como la adecuada red de apoyo que se explicará a continuación, son las principales razones por las cuales los participantes no reportaron las afectaciones al estado de ánimo asociadas a la larga trayectoria de la enfermedad - como señala la literatura - sino a etapas iniciales de la misma, cuando aún no habían incorporado esas estrategias.

En cuarto lugar, se describe el bienestar asociado al apoyo social de los participantes. Es particularmente relevante que la adaptación y comprensión ante los cambios en la vida sexual, por parte de sus parejas, fue descrito como uno de los determinantes de bienestar que influyen en la calidad de vida de los pacientes. De acuerdo con tres de los participantes, sentir la comprensión por parte de sus parejas y los cambios que se incorporan en la relación sexual mitigan en gran medida las afectaciones relacionadas con la función de dicho sistema. La literatura ${ }^{17}$ comúnmente reporta la función sexual como uno de los principales dominios afectados, principalmente por los sentimientos negativos asociados al deterioro en la función sexual y las implicaciones tanto personales como familiares que puede llegar a generar. La categoría de afectación a la calidad de vida relacionada con alteraciones en la función sexual es de las afectaciones más difíciles de enfrentar y de abordar con los pacientes. Así, la percepción del bienestar generado por la adaptación y comprensión ante los cambios en la vida sexual por parte de la pareja es un resultado importante, ya que incrementa la autoestima de los pacientes, y minimiza el impacto de esta alteración sobre la calidad de vida; por ello, puede orientar 
futuros abordajes para la intervención de dichas alteraciones en pro de la búsqueda de calidad de vida.

Por último, el apoyo familiar fue considerado crucial para mitigar las afectaciones causadas en todas las dimensiones, desde las alteraciones en el estado de ánimo hasta las afectaciones en la vida sexual o los síntomas generales del cáncer, por lo cual es vital tener en cuenta la red de apoyo de los pacientes de cara al afrontamiento de la enfermedad.

El estudio cualitativo descriptivo permitió explorar y describir cómo y por qué se afecta o se favorece la calidad de vida de los pacientes con este diagnóstico. El diseño elegido abordó la historia de salud y enfermedad centrada en los cambios y no las afectaciones, lo que permitió que se describieran las áreas que sufren mayor afectación en sus vidas, y que emergieran temas relacionados con las condiciones que incrementan la calidad de vida de los pacientes.

Este estudio fue desarrollado durante la emergencia del COVID-19, de manera que las limitaciones derivadas de la contingencia no permitieron acceder a un mayor número de participantes que ofrezcan mayor grado de variabilidad, principalmente en cuanto a las características de los participantes y el sistema de salud que les atiende. Por lo mismo, las entrevistas fueron realizadas de manera telefónica y no cara a cara, lo cual hubiera enriquecido la interacción, el rapport y la profundidad en el manejo de temas sensibles.

\section{Conclusiones}

La percepción de calidad de vida relacionada con la salud es un constructo compuesto de múltiples dimensiones, tanto físicas como emocionales, espirituales y sociales, en la que juegan un papel relevante la familia y el personal de salud. Los mecanismos de afrontamiento de cada individuo y la atención que recibe por parte del sistema de salud hacen parte también de esa multidimensionalidad; por ende, la evaluación que cada sujeto haga de su calidad de vida va a estar regida por cada una de estas dimensiones. El estudio descriptivo identifica el papel que puede jugar el sistema de salud en la calidad de vida del paciente, por lo que es importante avanzar en esta nueva línea de investigación. El fortalecimiento de la relación médico-paciente, basada en el trato cordial, disposición afectiva y empatía al momento de la valoración médica, se describe como una de las principales estrategias para incrementar la percepción de calidad de vida relacionada con la salud de los pacientes con cáncer de próstata avanzado. Lo anterior, sumado a la concientización del personal de salud con relación a los posibles síntomas que pueda presentar el paciente, los efectos adversos de/los tratamiento(s), así como el hecho de considerar en el acto médico el reconocimiento de las percepciones, sentimientos y temores que pueda llegar a estar sintiendo el paciente ante el doloroso proceso de enfermedad también va a favorecer mejores resultados en salud. Así mismo, el fortalecimiento de prácticas personales tales como la implementación de nuevos hábitos que promuevan la salud y el bienestar, entre ellos dieta, actividad física, actividades lúdicas y espiritualidad, entre otras, incrementa la capacidad de afrontamiento ante el nuevo proceso de enfermedad, mejora el estado de ánimo y, por ende, la percepción de calidad de vida relacionada con la salud. Por último, la consolidación de una adecuada red de soporte social como mecanismo de afrontamiento ante el proceso de enfermedad cobra vital importancia. El hecho de favorecer el acompañamiento de la familia y de su círculo social desde el inicio de la enfermedad es un generador de bienestar y de aumento en la percepción no sólo de la calidad de vida, sino del estado actual de salud.

\section{Financiación}

La presente investigación no ha recibido ninguna beca específica de agencias de los sectores público, comercial, o sin ánimo de lucro.

\section{Conflicto de Intereses}

Los autores no tienen conflicto de intereses que declarar.

\section{Referencias}

1 Cancer Research UK. Prostate Cancer Stat. Septiembre de 2014www.cancerresearchuk.org

2 International Agency for Research on Cancer. The Global Cancer Observatory, WHO. 2019www.gco.iarc.fr

3 Sellers L, Nuhoglu A, Davda R, Ricketts K, Payne H. Patientreported outcome measures in metastatic prostate cancer. Trends in Urology \& Men's Health 2016;7(01):28-32. Doi: $10.1002 /$ tre.504

4 Casey RG, Corcoran NM, Goldenberg SL. Quality of life issues in men undergoing androgen deprivation therapy: a review. Asian J Androl 2012;14(02):226-231. Doi: 10.1038/aja2011.108

5 Peralta L. La participación del paciente en la toma de decisiones en las consultas de atención primaria. Facultad de Medicina. Universidad de Cantabria. Tesis Doctoral, 2010. Available at: https://www. tdx.cat/bitstream/handle/10803/10705/TesisLPM.pdf

6 Wolf AM, Wender RC, Etzioni RB, et al; American Cancer Society Prostate Cancer Advisory Committee. American Cancer Society guideline for the early detection of prostate cancer: update 2010. CA Cancer J Clin 2010;60(02):70-98. Doi: 10.3322/caac.20066

7 Sandelowski M. Whatever happened to qualitative description? Res Nurs Health 2000;23(04):334-340. Doi: 10.1002/1098-240x (200008)23:4<334:aid-nur9>3.0.co;2-g

8 Umoquit MJ, Dobrow MJ, Lemieux-Charles L, Ritvo PG, Urbach DR, Wodchis WP. The efficiency and effectiveness of utilizing diagrams in interviews: an assessment of participatory diagramming and graphic elicitation. BMC Med Res Methodol 2008;8:53-65. Doi: 10.1186/1471-2288-8-53

9 Braun V, Clarke V. Using thematic analysis in psychology. Qual Res Psychol 2006;3(02):77-101. Doi: 10.1191/1478088706qp063oa

10 Mays N, Pope C. Qualitative research in health care. Assessing quality in qualitative research. BMJ 2000;320(7226):50-52. Doi: 10.1136/bmj.320.7226.50

11 Spall S. Peer Debriefing in Qualitative Research: Emerging Operational models. Qual Inq 1998;4(02):280-292. Doi: $10.1177 / 107780049800400208$

12 Guba G, Lincoln YS. Effective evaluation: improving the usefulness of evaluation results through responsive and naturalistic approaches. J Higher Educ 1983;54(03):339-342. Doi: $10.2307 / 1981810$

$13 \mathrm{Kim} \mathrm{H}$, Sefcik JS, Bradway C. Characteristics of Qualitative Descriptive Studies: A Systematic Review. Res Nurs Health 2017;40(01):23-42. Doi: 10.1002/nur.21768 


\section{Percepción de la calidad de vida en pacientes con cáncer de próstata avanzado Parra-Morales et al.}

14 Fielding N, Lee R, Blank G. The SAGE Handbook of Online Research Methods. SAGE Publications Ltd.; 2008

15 Reyes D, González J, Mohar A, Meneses A. Epidemiología del dolor por cáncer. Rev Soc Esp Dolor 2011;18:118-134 ISSN 1134-8046

16 Gonzalez Barón M, Rodriguez M, Ordoñez, Gallego A. La astenia en el paciente con cáncer. Rev Oncológica 2001;3:292-299. Doi: $10.1007 / \mathrm{BF} 02718421$

17 Wilde K, Cruz C, Rojas L, Novoa M. Efectos secundarios del tratamiento de cáncer de próstata localizado, en calidad de vida y ajuste marital. Urol Colomb. 2020;7-19. ISSN 1657-9267

18 Orsola A, Morote J. Epidemiología de la incontinencia urinaria en el cáncer de próstata. Incidencia, calidad de vida y aspectos farmacoeconómicos. Arch Esp Urol 2009;62(10):786-792. Doi: 10.0000/scielo.isciii.es/S0004-06142009001000003

19 Ramirez J, Castro D, Lerma C, Yela F, Escobar F. Consecuencias de la pandemia COVID 19 en la salud mental asociadas al aislamiento social. Universidad Nacional de Colombia. Preprint Scielo. 2020. Available at: https://www.researchgate.net/publication/341116487_ CONSECUENCIAS_DE_LA_PANDEMIA_COVID_19_EN_LA_SALUD_ MENTAL_ASOCIADAS_AL_AISLAMIENTO_SOCIAL Doi: 10.1590/SciELO Preprints.303
20 Vicente J, Lopez A. Cáncer en población trabajadora. Incapacidad y riesgo de exclusión laboral y social. Med Segur Trab 2018;64 (253):354-78 ISSN 1989-7790

21 Giraldo C, Ceballos G. Acostumbrarse a las barreras: Estudio cualitativo de las barreras del sistema de salud colombiano para el diagnóstico y tratamiento oportuno de cáncer de mama. Forum: Qualitative Social Research 2011;12(02):5. Doi: $10.17169 /$ fqs-12.2.1650

22 Schwartzmann L. Calidad de vida relacionada con la salud: Aspectos Conceptuales. Cienc Enferm 2003;9(02):9-21. Doi: 10.4067/S0717-95532003000200002

23 Quintana López VA, Díaz López KJ, Caire Juvera G. Intervenciones para promover estilos de vida saludables y su efecto en las variables psicológicas en sobrevivientes de cáncer de mama: revisión sistemática. Nutr Hosp 2018;35(04):979-992. Doi: 10.20960/nh.1680

24 Eller LS, Lev EL, Gejerman G, et al. Prospective study of quality of life of patients receiving treatment for prostate cancer. Nurs Res 2006; 55(2, Suppl):S28-S36. Doi: 10.1097/00006199-20060300100006 